CORRIGENDUM

\title{
South Africa: from species cradle to genomic applications
}

Billie-Jo Hardy, Béatrice Séguin, Raj Ramesar, Peter A. Singer and Abdallah S. Daar

Nature Reviews Genetics 9 (Suppl.), S19-S23 (2008).

On page S21 of this Perspective under the 'Local health benefits' subheading, the following text was incorrect:

Another major project is a public-private admixture mapping study recently initiated between researchers at the Centre of Excellence for Biomedical TB Research and GlaxoSmithKline (with additional funding from the Wellcome Trust and the University of Oxford). The study involves up to 1,000 samples from the South African Coloured population. Previous genome-wide scans and genetic variation studies in this population have contributed to increasing understanding of genetic susceptibility to $\mathrm{TB}^{15,16,17}$, including revealing the novel target loci MC $3 R$ (melanocortin 3 receptor) and CTSZ (cathepsin Z) ${ }^{17}$. It is hoped that the data generated by the admixture study will make similar contributions. Confirming the limited local capacity faced by South African researchers when conducting scaled-up human genomic variation studies, the admixture-mapping study samples were exported and the data generation and preliminary analysis was conducted at the Affymetrix laboratories in the United States.

The text should have read:

Another major project is a public-private admixture mapping study recently initiated between researchers at the DST/NRF Centre of Excellence for Biomedical TB Research at Stellenbosch University and GlaxoSmithKline. The study involves up to 1,000 samples from the South African Coloured population. Previous genome-wide scans and genetic variation studies in this population have contributed to increasing understanding of genetic susceptibility to $\mathrm{TB}^{15,16,17}$, including revealing novel target loci MC3R (melanocortin 3 receptor) and CTSZ (cathepsin Z) ${ }^{17}$. It is hoped that the data generated by the admixture study will make similar contributions. Confirming the limited local capacity faced by South African researchers when conducting scaled-up human genomic variation studies, the admixture-mapping study samples were analysed by SNP chip technology and the data generated at the Affymetrix laboratories in the United States. Raw data was provided to Stellenbosch University.

The authors apologize for this error. 